\title{
Ameliorating effect of luteolin on memory impairment in an Alzheimer's disease model
}

\author{
HUIMIN WANG ${ }^{1}$, HUILING WANG ${ }^{2}$, HUIXIN CHENG $^{3}$ and ZHENYONG CHE ${ }^{1}$ \\ ${ }^{1}$ Department of Neurology, Xinxiang Central Hospital; ${ }^{2}$ Department of Pediatrics, Xinxiang Hospital of \\ Municipal Offices; ${ }^{3}$ Department of Pathology, Xinxiang Central Hospital, Xinxiang, Henan 453000, P.R. China
}

Received November 24, 2015; Accepted March 28, 2016

DOI: $10.3892 / \mathrm{mmr} .2016 .5052$

\begin{abstract}
Alzheimer's disease (AD) is one of the most prevalent neurodegenerative disorder. It is characterized by the formation of amyloid plaques and neurofibrillary tangles in the brain, the degeneration of cholinergic neurons and neuronal cell death. The present study aimed to investigate the effect of luteolin, a flavonoid compound, on memory impairment in a streptozotocin (STZ)-induced Alzheimer's rat model. Morris water maze and probe tests were performed to examine the effect of luteolin treatment on cognition and memory. The effect of luteolin on CA1 pyramidal layer thickness was also examined. The results demonstrated that luteolin significantly ameliorated the spatial learning and memory impairment induced by STZ treatment. STZ significantly reduced the thickness of CA1 pyramidal layer and treatment of luteolin completely abolished the inhibitory effect of STZ. Our results suggest that luteolin has a potentially protective effect on learning defects and hippocampal structures in AD.
\end{abstract}

\section{Introduction}

Alzheimer's disease (AD), a progressive neurodegenerative disease of the central nervous system, is the most common cause of senile dementia (1). AD is characterized by irreversible loss of neurons and a clinically gradual deterioration in intellectual abilities including cognition and memory (1). The $\mathrm{AD}$ brain is characterized by the presence of neuritic plaques, neurofibrillary tangles, and the loss of cortical neurons and synapses (2). $\beta$-amyloid $(\mathrm{A} \beta)$, generated from a sequent cleavage of amyloid precursor protein (APP) by $\beta$-site APP cleavage enzyme 1 and $\gamma$-secretase, is the major component of extracellular neuritic plaques (3). The accumulation of $\mathrm{A} \beta$

Correspondence to: Dr Zhenyong Che, Department of Neurology, Xinxiang Central Hospital, 56 Jinsui Road, Xinxiang, Henan 453000, P.R. China

E-mail: zhenyongche@163.com

Key words: Alzheimer's disease, Morris water maze test, luteolin contributes to $\mathrm{AD}$ pathogenesis in that the accumulation of $\mathrm{A} \beta$ induces an increase in intracellular reactive oxygen species (ROS) and neuronal cell apoptosis (4-7). Previous findings emphasize the critical role of oxidative stress as a major cause in the pathogenesis of $\mathrm{AD}(8,9)$. Therefore, antioxidants may attenuate $\mathrm{A} \beta$-induced neurotoxicity and cell death, leading to the amelioration of AD-induced impairment in cognition and memory $(10,11)$.

Flavonoids have a 15-carbon skeleton composed of two fused 6-membered rings (an aromatic ring and a heterocyclic ring) connected through a carbon-carbon bridge to an aromatic ring (12). In the human diet, flavonoids constitute the most common group of polyphenolic compounds that are synthesized in plant cells. The phenolic hydroxyl groups attached to ring structures of flavonoids allow them to act as antioxidants $(13,14)$, as radical-scavenging agents $(15,16)$, or as agents of anti-inflammation $(17,18)$. Accumulating evidence showed that the consumption of flavonoid-rich foods is associated with lower rates of dementia and has some beneficial effects on memory and learning $(19,20)$.

Luteolin, a flavonoid compound, has been suggested to have neuroprotective properties. Findings of in vivo studies examining rat models have shown that luteolin protects against cognitive dysfunction induced by chronic cerebral hypoperfusion (21). Luteolin also protects against high-fat diet-induced cognitive defects in obesity mice (22). Fu et al (21) recently reported that luteolin can protect against cognitive dysfunction induced by chronic cerebral hypoperfusion in rats. In the context of AD, Rezai-Zadeh et al (23) showed that luteolin treatment of murine N2a cells transfected with SweAPP and primary neuronal cells derived from SweAPP-overexpressing mice resulted in a significant reduction in $A \beta$ generation. The mechanism may involve selective inactivation of the GSK-3 $\alpha$ isoform, which increases the phosphorylation of PS1, the catalytic core of the $\gamma$-secretase complex, thereby reducing PS1-APP interaction and A $\beta$ generation. In a later study, Zhou et al (24) reported that luteolin attenuated zinc-induced hyperphosphorylation of the protein $\tau$ in SH-SY5Y cells through its antioxidant action. Luteolin also inhibited the $\tau$ kinase p7056K but established recovery of total phosphatase activity. More recently, it was found that luteolin reduced AD pathologies induced by traumatic brain injury (25). However, the overall neuroprotective effect of luteolin in drug-induced Alzheimer's rat models remains to be investigated. The present 
study aimed to investigate the effect of luteolin on spatial learning and the structure of CA1 pyramidal layer thickness in a streptozotocin (STZ)-induced AD model.

\section{Materials and methods}

Animals. A total of 60 male Wistar rats, weighing 200-230 g, and aged 3 months, were housed in cages with a temperature of $24-26^{\circ} \mathrm{C}$ and a 12 -h dark/light cycle with food and water ad libitum. Behavioral experiments were performed in the morning. The experiment protocols were approved by the Animal Ethics Committee of the Xinxiang Central Hospital (Henan, China).

Establishment of the animal model. Animals were anesthetized with ketamine $(80 \mathrm{mg} / \mathrm{kg})$ and xylazine $(15 \mathrm{mg} / \mathrm{kg})$ intra-peritoneally, and their heads were fixed into a stereotaxic instrument (Narishige, Tokyo, Japan). Two stainless 23-gauge guide cannula were implanted in the lateral ventricles bilaterally. Stereotaxic coordinates were based on Paxinos and Watson atlas of the rat brain. Following surgery, the animals were kept in cages for 6 days to recover.

Experimental protocol. The 60 rats were divided into 6 groups with 10 rats in each group. Apart from the control group in which the rats did not receive any treatment or surgery, the rats in the remaining 5 groups received surgery and recovery treatments. In the saline group, rats received normal saline after recovery; in the sham group, rats received $10 \%$ DMSO after recovery; in the STZ group (Alzheimer's model), rats received STZ $(3 \mathrm{mg} / \mathrm{kg})$ on the fourth and sixth days after recovery; and in the L10 and L20 groups, STZ was injected on days 4 and 6 and the rats were treated with luteolin at doses of 10 and $20 \mathrm{mg} / \mathrm{kg}$, respectively, on the 1st, 2nd, 3rd and 5th days after recovery.

Drugs were injected via the intracerebroventricular (ICV) route in a total volume of $10 \mu \mathrm{l}$ at the rate of $1 \mu \mathrm{l} / \mathrm{min}$. The 27 -gauge injection needle was inserted into the guide cannula. The injection needle was attached to a $10 \mu \mathrm{l}$ syringe by a polyethylene tube.

Morris water maze test. The Morris water maze consists of a circular water tank with $160 \mathrm{~cm}$ diameter and $60 \mathrm{~cm}$ height, filled with non-toxic water $\left(25 \pm 2^{\circ} \mathrm{C}\right)$ to a depth of $25 \mathrm{~cm}$. The pool was divided into 4 quadrants (North, South, East and West) which were used as start points. An escape platform (10 $\mathrm{cm}$ in diameter) made of plexiglass was placed in the middle of one of the randomly selected quadrants of the pool, $1 \mathrm{~cm}$ below the surface of water and kept in the same position throughout the entire experiment (North-West for this study). Spatial learning of animals was tested 14 days after STZ infusion in the Morris water maze. The rats in each group were tested (one at a time). The rats were trained for four days prior to the formal experiments. Each rat was subjected to 4 consecutive trials on each day with an interval of $1 \mathrm{~min}$.

Each trial was initiated by placing the rat randomly at 1 of the 4 starting points. The rats were allowed to swim in the pool during a period of $90 \mathrm{sec}$ to locate the hidden platform. If a rat did not locate the hidden platform within this period, it was manually guided to the platform by the investigator. The rats were allowed to remain on the platform for $30 \mathrm{sec}$. All the trials were performed at 9 a.m.

Directions of the rats were recorded by a video camera (Fuji, Tokyo, Japan) above the center of the maze that was linked to a computer. Spatial acquisition was evaluated by measuring escape latency (time to find the platform), traveled distance (path length to reach the platform), and swimming speed using the EthoVision tracking system (Noldus Information Technology, Wageningen, The Netherlands), as described previously $(26,27)$. The data obtained from rats with visual impairment were excluded.

Probe test. To assess memory consolidation, a probe test was performed $24 \mathrm{~h}$ after the Morris water maze test. For the probe test, the platform was removed and the rats were allowed to swim freely. The swimming pattern of every rat was recorded with a camera. Consolidated spatial memory was estimated by the time spent in the target quadrant area.

Tissue preparation. The rats were anesthetized with Zoletil 50 $(10 \mathrm{mg} / \mathrm{kg}$, i.m.) and perfused intra-cardiac infusion with $100 \mathrm{mmol} / \mathrm{l}$ phosphate-buffered saline (PBS) followed by ice-cold $4 \%$ paraformaldehyde. The brains of the rats were isolated and post-fixed in $50 \mathrm{mmol} / \mathrm{l} \mathrm{PBS}$ containing $4 \%$ paraformaldehyde overnight, immersed in a solution containing $30 \%$ sucrose in $50 \mathrm{mmol} / 1 \mathrm{PBS}$ and stored at $4^{\circ} \mathrm{C}$ until sectioning. The frozen brains were coronally sectioned on paraffin at $3 \mu \mathrm{m}$ and then stored in a storage solution at $4^{\circ} \mathrm{C}$.

Immunohistochemistry. For the evaluation of dendritic damage, the sections were immunostained with a rabbit antibody against rat MAP2 (Millipore Corp., Billerica, MA, USA) at a 1:250 dilution. Three sections from each animal were assessed for scoring.

Statistical analysis. SPSS 13.0 software (SPSS Inc., Chicago, IL, USA) was used for data analysis. Analysis of variance was used for comparison of the behavioral and histological data. A Tukey multiple comparison post-test was performed to assess differences between groups. $\mathrm{P}<0.05$ was considered to indicate a statistically significant.

\section{Results}

Luteolin improved the cognitive function of rats with STZ in Morris water maze test. To evaluate the cognitive function of rats, the Morris water maze test was performed. The mean escape latency of rats in different groups did not show a significant difference for the first day $(\mathrm{P}>0.05)$. However, there was a significant difference in escape latency from the second day $(\mathrm{P}<0.05)$. The rats treated with STZ showed a significantly decreased ability to locate the platform and to learn its location on the fifth day of training (Fig. 1A). By contrast, this poor performance was significantly mitigated by the treatment with luteolin (10 and $20 \mathrm{mg} / \mathrm{kg}$ ) as shown by the decreased latency to locate the platform from the second day of training. The effect of treatment with luteolin at 10 and $20 \mathrm{mg} / \mathrm{kg}$ was not significantly different $(\mathrm{P}>0.05)$.

Luteolin decreased the travel distance to reach the hidden platform (path length). Progressive decreases in the path 

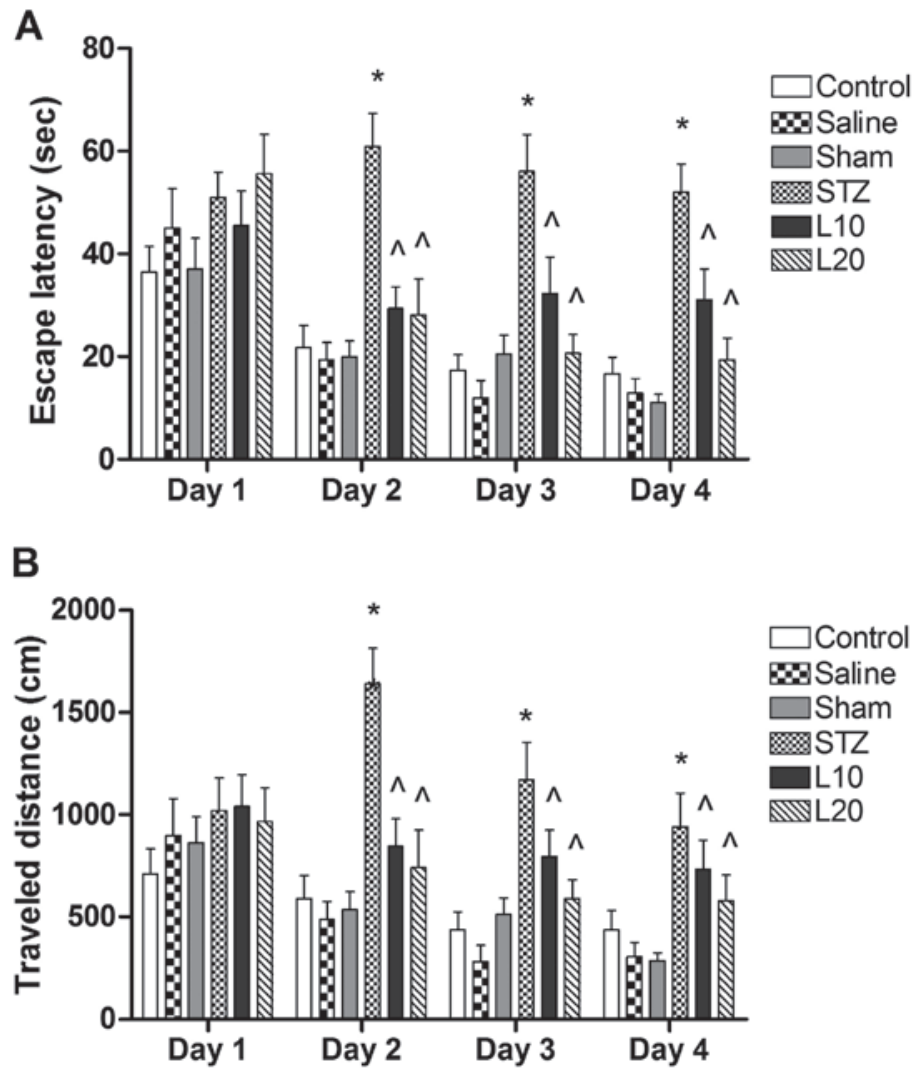

Figure 1. Effect of luteolin on (A) escape latency and (B) traveled distance of the streptozotocin (STZ)-induced Alzheimer's disease rat model. "P<0.05 vs. control, ${ }^{\wedge} \mathrm{P}<0.05$ vs. $\mathrm{STZ}$ group.

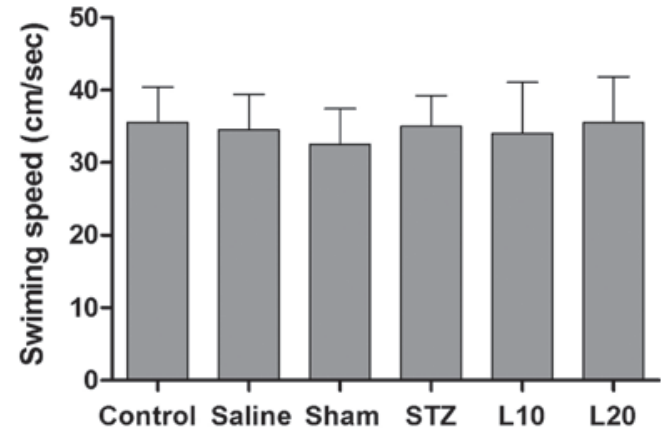

Figure 2. Effect of luteolin on swimming speed of the Alzheimer's disease rat model. STZ, streptozotocin.

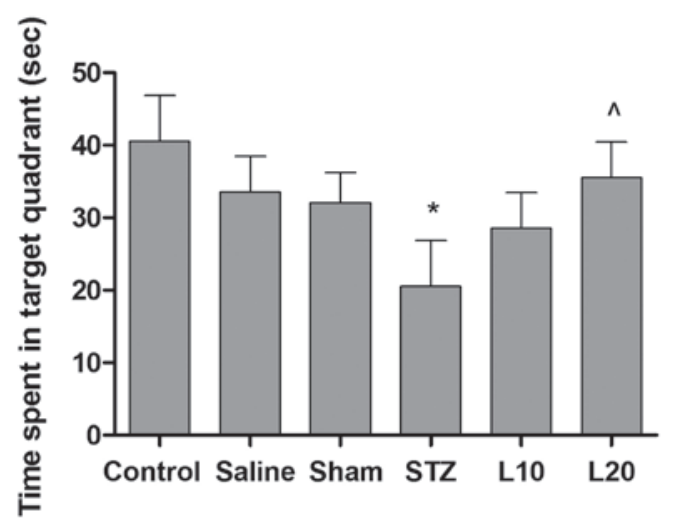

Figure 3. Effect of luteolin on performance in probe test of the Alzheimer's disease rat model. ${ }^{*} \mathrm{P}<0.05$ vs. control, ${ }^{\wedge} \mathrm{P}<0.05$ vs. streptozotocin (STZ) group. length to reach the hidden platform on subsequent days in the water maze task were associated with intact memory of animals. Therefore, the traveled distance was measured to evaluate the memory impairment. As shown in Fig. 1B, the total distance traveled to reach the hidden platform did not differ between any of the groups on the first day of testing in the Morris water maze. However, there was a significant difference in the traveled distance of STZ-treated rats as compared to the sham animals from the second day onwards $(\mathrm{P}<0.05)$. Chronic treatment with luteolin $(10$ and $20 \mathrm{mg} / \mathrm{kg})$ significantly $(\mathrm{P}<0.05)$ decreased the total distance traveled to reach the platform in the STZ-injected rats, which suggested improvement in memory associated with luteolin treatment. When the effect of luteolin at different doses was compared, the reduction in traveled distance with luteolin at doses of 10 and $20 \mathrm{mg} / \mathrm{kg}$ was not significantly different $(\mathrm{P}>0.05)$.

Luteolin has no effect on swimming speed of ICV-STZ-injected rats. As shown in Fig. 2, no significant difference in swimming speed was observed between any groups in the probe trial. The mean values in control, saline, sham, STZ, and luteolin $(10$ and $20 \mathrm{mg} / \mathrm{kg}$ ) groups were $35.5 \pm 3.7,34.6 \pm 5.1,32.7 \pm 3.6$, $35.6 \pm 4.6,34.5 \pm 3.9$ and $35.7 \pm 4.9 \mathrm{~cm} / \mathrm{sec}$, respectively $(\mathrm{P}>0.05)$, indicating that there was no motor activity disturbances in tested animals.

Effect of luteolin on time spent in target quadrant in $I C V$-STZ-injected rats. To examine the learning ability and consolidation of the platform location during the training, a probe test was performed. As shown in Fig. 3, animals in 

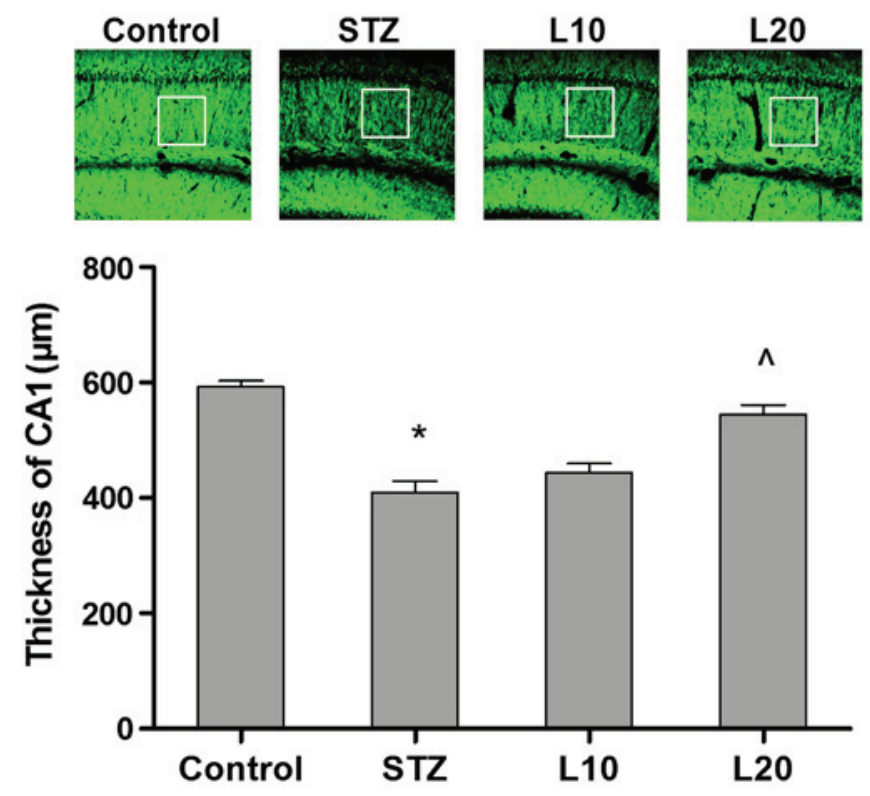

Figure 4. Effect of luteolin on CA1 pyramidal layer thickness of the Alzheimer's disease rat model. ${ }^{*} \mathrm{P}<0.05$ vs. control, ${ }^{\wedge} \mathrm{P}<0.05$ vs. streptozotocin (STZ) group.

different groups showed a significant different performance. The time spent in the target quadrant was significantly lower in the STZ rats as compared to the sham group. STZ rats treated with luteolin (10 and $20 \mathrm{mg} / \mathrm{kg}$ ) spent more time in the target quadrant than the monotherapy group in the probe test. In particular, STZ-treated rats with luteolin at a dose of $20 \mathrm{mg} / \mathrm{kg}$ spent significantly more time in the target quadrant compared with STZ-treated rats $(\mathrm{P}<0.05)$.

Luteolin increased the thickness of CA1 pyramidal layer. The thickness of the hippocampal CA1 pyramidal layer in the tested groups was measured to test the effect of luteolin treatment. The mean CA1 pyramidal layer thicknesses were $592.5 \pm 27.2 \mu \mathrm{m}$ in the control group and $409.3 \pm 23.6 \mu \mathrm{m}$ in the STZ group $(\mathrm{P}<0.05)$. The mean CA1 pyramidal layer thickness in the L20 group was $544.3 \pm 32.6 \mu \mathrm{m}$ showing a significant increase compared to $409.3 \pm 23.6 \mu \mathrm{m}$ of the STZ group ( $\mathrm{P}<0.05$, Fig. 4).

\section{Discussion}

Flos Chrysanthemi, the flower of Dendranthema morifolium Ramat Tzvel (Chrysanthemum morifolium Ramat.), is known as Ju-Hua in Chinese. This herb is listed in Shen Nong's Herbal (a historical book of Chinese herbs) as a non-toxic, top-grade herb that has been used as an agent for the treatment of headache, vertigo, and sore throat (28). Flos Chrysanthemi is an edible medicinal herb, and one of the major active ingredients of this plant is luteolin, a flavonoid compound. The pharmacological activity of luteolin is thought to be associated in part with its antioxidant potential $(28,29)$, anti-tumorigenic effects $(30,31)$, and anti-inflammatory/anti-allergic activities (29). In addition, it has been reported that luteolin can act as an inhibitor of protein kinase $\mathrm{C}$ and lipoxygenase (32). In vitro and in vivo studies have also shown that luteolin reduces high blood cholesterol through the inhibition of cholesterol uptake in Caco-2 cells and affects cholesterol transport (33).
Luteolin also has a radio-protective and a protective effect on doxorubicin-induced cardiotoxicity in mice $(28,34)$. The results of the present study indicate that luteolin reduced the escape latency and traveled distance parameters in the Morris water maze while increasing the time spent in the target quadrant in the animal model of AD. These results indicate that luteolin can improve spatial learning and memory impairment in this experimental model. It also prevents the thickness reduction of the CA1 pyramidal cell layer. This finding along with the previous in vitro studies revealed the neuroprotective effect of triazine derivatives in the experimental model $(23,24)$.

The ICV STZ model produces cognitive defects similar to those observed in the sporadic dementia of Alzheimer's type (35). STZ administration in rats induces oxidative stress in the brain, $A \beta$ plaques aggregation, $\tau$ protein hyperphosphorylation, neuroinflammation, and apoptosis, which cooperate to repair memory and learning in the AD animal models $(35,36)$. A $\beta$ plaque is the proteolytic product of APP, which can generate the ROS, especially hydrogen peroxides, leading to cell death in the neuronal cultures and toxicity in hippocampal neurons (37-39). On the other hand, STZ (ICV-STZ) in sub-diabetogenic dose reduces energy metabolism, leading to cognitive dysfunction by inhibiting the synthesis of adenosine triphosphate. The repair of glucose and energy impairment caused by STZ is a potential source for this oxidative stress (40). Oxidative stress is the most important hypothesis involved in the pathophysiology of AD in that excess free radicals of oxygen lead to cell damage, a progressive cognition and memory loss $(38,39,41)$. Additionally, the reciprocal effects of oxidative stress and $A \beta$ aggregation intensify the impairment of neurological function (5). In the present study, the results showed that STZ injection induced significant defects of memory, indicating the success of AD model establishment.

Given the critical role of ROS in the pathogenesis of AD, numerous ROS scavengers have been employed to investigate whether they can protect against memory 
defects in an experimental model of AD $(42,43)$. A number of ROS modulators including flavonoid compounds such as EGCG attenuate cell injury and neurotoxicity during $\mathrm{A} \beta$ exposure (44). Accumulating evidence has shown that luteolin can act as a ROS scavenger agent in a number of tissues or cells. Yu et al (45) reported that luteolin can protect myocardial ischemia/reperfusion injury by inhibiting the ROS-MAPK-mediated mechanism. Another study showed that luteolin inhibits $\mathrm{Cr}$ (VI)-induced malignant cell transformation of human lung epithelial cells by targeting ROS-mediated multiple cell signaling pathways (46). In human umbilical vein endothelial cells, luteolin suppressed the TNF- $\alpha$-activated ROS generation, and the expression of Nox4, p22phox, and ICAM-1 and VCAM-1 (47). The present findings suggest that luteolin improved memory impairment and prevents the reduction of CA1 pyramidal cell layer in an AD model. Thus, the neuroprotective effect of luteolin against $\mathrm{AD}$ nay be due to the antioxidative characteristic of this agent by inhibiting free radical products and dispersing $\mathrm{A} \beta$ plaques. Therefore, luteolin is potentially a suitable therapeutic candidate for neural disorders such as AD, although further studies including clinical trials, are needed to confirm this hypothesis.

\section{References}

1. Zhu Z, Yan J, Jiang W, Yao XG, Chen J, Chen L, Li C, Hu $\mathrm{L}$, Jiang $\mathrm{H}$ and Shen $\mathrm{X}$ : Arctigenin effectively ameliorates memory impairment in Alzheimer's disease model mice targeting both $\beta$-amyloid production and clearance. J Neurosci 33: 13138-13149, 2013.

2. Terry RD, Masliah E, Salmon DP, Butters N, DeTeresa R, Hill R, Hansen LA and Katzman R: Physical basis of cognitive alterations in Alzheimer's disease: synapse loss is the major correlate of cognitive impairment. Ann Neurol 30: 572-580, 1991.

3. Choi SM, Kim BC, Cho YH, Choi KH, Chang J, Park MS, Kim MK, Cho KH and Kim JK: Effects of flavonoid compounds on $\beta$-amyloid-peptide-induced neuronal death in cultured mouse cortical neurons. Chonnam Med J 50: 45-51, 2014.

4. Marcus DL, Thomas C, Rodriguez C, Simberkoff K, Tsai JS, Strafaci JA and Freedman ML: Increased peroxidation and reduced antioxidant enzyme activity in Alzheimer's disease. Exp Neurol 150: 40-44, 1998.

5. Zheng L, Kågedal K, Dehvari N, Benedikz E, Cowburn R, Marcusson J and Terman A: Oxidative stress induces macroautophagy of amyloid beta-protein and ensuing apoptosis. Free Radic Biol Med 46: 422-429, 2009.

6. Behl C and Holsboer F: Oxidative stress in the pathogenesis of Alzheimer's disease and antioxidant neuroprotection. Fortschr Neurol Psychiatr 66: 113-121, 1998 (In German).

7. Peng QL, Buz'Zard AR and Lau BH: Pycnogenol protects neurons from amyloid-beta peptide-induced apoptosis. Brain Res Mol Brain Res 104: 55-65, 2002.

8. Andersen JK: Oxidative stress in neurodegeneration: cause or consequence? Nat Med 10 Suppl: S18-S25, 2004.

9. Butterfield DA, Perluigi M and Sultana R: Oxidative stress in Alzheimer's disease brain: New insights from redox proteomics. Eur J Pharmacol 545: 39-50, 2006.

10. Aliev G, Obrenovich ME, Reddy VP, Shenk JC, Moreira PI, Nunomura A, Zhu X, Smith MA and Perry G: Antioxidant therapy in Alzheimer's disease: theory and practice. Mini Rev Med Chem 8: 1395-1406, 2008.

11. Lin YH, Liu AH, Wu HL, Westenbroek C, Song QL, Yu HM, Ter Horst GJ and Li XJ: Salvianolic acid B, an antioxidant from Salvia miltiorrhiza, prevents Abeta(25-35)-induced reduction in BPRP in PC12 cells. Biochem Biophys Res Commun 348: 593-599, 2006.

12. Merken HM and Beecher GR: Measurement of food flavonoids by high-performance liquid chromatography: A review. J Agric Food Chem 48: 577-599, 2000.
13. Rice-Evans CA, Miller NJ and Paganga G: Structure-antioxidant activity relationships of flavonoids and phenolic acids. Free Radic Biol Med 20: 933-956, 1996.

14. Zeng LH, Zhang HD, Xu CJ, Bian YJ, Xu XJ, Xie QM and Zhang RH: Neuroprotective effects of flavonoids extracted from licorice on kainate-induced seizure in mice through their antioxidant properties. J Zhejiang Univ Sci B 14: 1004-1012, 2013.

15. Dugas AJ Jr, Castañeda-Acosta J, Bonin GC, Price KL, Fischer NH and Winston GW: Evaluation of the total peroxyl radical-scavenging capacity of flavonoids: Structure-activity relationships. J Nat Prod 63: 327-331, 2000.

16. Cho JG, Song NY, Nam TG, Shrestha S, Park HJ, Lyu HN, Kim DO, Lee G, Woo YM, Jeong TS, et al: Flavonoids from the grains of $\mathrm{C} 1 / \mathrm{R}-\mathrm{S}$ transgenic rice, the transgenic Oryza sativa spp. japonica, and their radical scavenging activities. J Agric Food Chem 61: 10354-10359, 2013.

17. Nijveldt RJ, van Nood E, van Hoorn DE, Boelens PG, van Norren $\mathrm{K}$ and van Leeuwen PA: Flavonoids: a review of probable mechanisms of action and potential applications. Am J Clin Nutr 74: 418-425, 2001.

18. Thilakarathna SH and Rupasinghe HP: Flavonoid bioavailability and attempts for bioavailability enhancement. Nutrients 5: 3367-3387, 2013.

19. Beking K and Vieira A: Flavonoid intake and disability-adjusted life years due to Alzheimer's and related dementias: a population-based study involving twenty-three developed countries. Public Health Nutr 13: 1403-1409, 2010.

20. Letenneur L, Proust-Lima C, Le Gouge A, Dartigues JF and Barberger-Gateau P: Flavonoid intake and cognitive decline over a 10-year period. Am J Epidemiol 165: 1364-1371, 2007.

21. Fu X, Zhang J, Guo L, Xu Y, Sun L, Wang S, Feng Y, Gou L, Zhang L and Liu Y: Protective role of luteolin against cognitive dysfunction induced by chronic cerebral hypoperfusion in rats. Pharmacol Biochem Behav 126: 122-130, 2014.

22. Liu Y, Fu X, Lan N, Li S, Zhang J, Wang S, Li C, Shang Y, Huang $\mathrm{T}$ and Zhang L: Luteolin protects against high fat diet-induced cognitive deficits in obesity mice. Behav Brain Res 267: 178-188, 2014.

23. Rezai-Zadeh K, Douglas Shytle R, Bai Y, Tian J, Hou H, Mori T, Zeng J, Obregon D, Town T and Tan J: Flavonoid-mediated presenilin-1 phosphorylation reduces Alzheimer's disease beta-amyloid production. J Cell Mol Med 13: 574-588, 2009.

24. Zhou F, Chen S, Xiong J, Li Y and Qu L: Luteolin reduces zinc-induced tau phosphorylation at Ser262/356 in an ROS-dependent manner in SH-SY5Y cells. Biol Trace Elem Res 149: 273-279, 2012.

25. Sawmiller D, Li S, Shahaduzzaman M, Smith AJ, Obregon D, Giunta B, Borlongan CV, Sanberg PR and Tan J: Luteolin reduces Alzheimer's disease pathologies induced by traumatic brain injury. Int J Mol Sci 15: 895-904, 2014.

26. Sharifzadeh M, Naghdi N, Khosrovani S, Ostad SN, Sharifzadeh K and Roghani A: Post-training intrahippocampal infusion of the COX-2 inhibitor celecoxib impaired spatial memory retention in rats. Eur J Pharmacol 511: 159-166, 2005.

27. Tabrizian K, Najafi S, Belaran M, Hosseini-Sharifabad A, Azami K, Hosseini A, Soodi M, Kazemi A, Abbas A and Sharifzadeh M: Effects of selective iNOS inhibitor on spatial memory in recovered and non-recovered ketamine induced-anesthesia in wistar rats. Iran J Pharm Res 9: 313-320, 2010.

28. Shimoi K, Masuda S, Furugori M, Esaki S and Kinae N: Radioprotective effect of antioxidative flavonoids in gamma-ray irradiated mice. Carcinogenesis 15: 2669-2672, 1994.

29. Seelinger G, Merfort I and Schempp CM: Anti-oxidant, anti-inflammatory and anti-allergic activities of luteolin. Planta Med 74: 1667-1677, 2008.

30. Yasukawa K, Takido M, Takeuchi $M$ and Nakagawa S: Effect of chemical constituents from plants on 12-O-tetradecanoylphorbol-13-acetate-induced inflammation in mice. Chem Pharm Bull (Tokyo) 37: 1071-1073, 1989.

31. Majumdar D, Jung KH, Zhang H, Nannapaneni S, Wang $X$, Amin AR, Chen Z, Chen ZG and Shin DM: Luteolin nanoparticle in chemoprevention: in vitro and in vivo anticancer activity. Cancer Prev Res (Phila) 7: 65-73, 2014.

32. Ferriola PC, Cody V and Middleton E Jr: Protein kinase C inhibition by plant flavonoids. Kinetic mechanisms and structure-activity relationships. Biochem Pharmacol 38: $1617-1624,1989$. 
33. Nekohashi M, Ogawa M, Ogihara T, Nakazawa K, Kato $\mathrm{H}$, Misaka T, Abe K and Kobayashi S: Luteolin and quercetin affect the cholesterol absorption mediated by epithelial cholesterol transporter niemann-pick c1-like 1 in caco-2 cells and rats. PLoS One 9: e97901, 2014.

34. Bast A, Kaiserová H, den Hartog GJ, Haenen GR and van der Vijgh WJ: Protectors against doxorubicin-induced cardiotoxicity: Flavonoids. Cell Biol Toxicol 23: 39-47, 2007.

35. Salkovic-Petrisic $M$ and Hoyer S: Central insulin resistance as a trigger for sporadic Alzheimer-like pathology: an experimental approach. J Neural Transm Suppl 72: 217-233, 2007.

36. Grünblatt E, Salkovic-Petrisic M, Osmanovic J, Riederer P and Hoyer S: Brain insulin system dysfunction in streptozotocin intracerebroventricularly treated rats generates hyperphosphorylated tau protein. J Neurochem 101: 757-770, 2007.

37. Maiese $\mathrm{K}$ and Chong ZZ: Insights into oxidative stress and potential novel therapeutic targets for Alzheimer disease. Restor Neurol Neurosci 22: 87-104, 2004.

38. Behl C, Davis JB, Lesley R and Schubert D: Hydrogen peroxide mediates amyloid beta protein toxicity. Cell 77: 817-827, 1994.

39. Varadarajan S, Yatin S, Kanski J, Jahanshahi F and Butterfield DA: Methionine residue 35 is important in amyloid beta-peptide-associated free radical oxidative stress. Brain Res Bull 50: 133-141, 1999.

40. Sharma M and Gupta YK: Intracerebroventricular injection of streptozotocin in rats produces both oxidative stress in the brain and cognitive impairment. Life Sci 68: 1021-1029, 2001.

41. Selkoe DJ: Alzheimer's disease: Genes, proteins, and therapy. Physiol Rev 81: 741-766, 2001.
42. Rasoolijazi H, Joghataie MT, Roghani M and Nobakht M: The beneficial effect of (-)-epigallocatechin-3-gallate in an experimental model of Alzheimer's disease in rat: a behavioral analysis. Iran Biomed J 11: 237-243, 2007.

43. Gilgun-Sherki Y, Rosenbaum Z, Melamed E and Offen D Antioxidant therapy in acute central nervous system injury: current state. Pharmacol Rev 54: 271-284, 2002.

44. Subramaniam R, Koppal T, Green M, Yatin S, Jordan B, Drake J and Butterfield DA: The free radical antioxidant vitamin $\mathrm{E}$ protects cortical synaptosomal membranes from amyloid beta-peptide(25-35) toxicity but not from hydroxynonenal toxicity: relevance to the free radical hypothesis of Alzheimer's disease. Neurochem Res 23: 1403-1410, 1998.

45. Yu D, Li M, Tian Y, Liu J and Shang J: Luteolin inhibits ROS-activated MAPK pathway in myocardial ischemia/reperfusion injury. Life Sci 122: 15-25, 2015.

46. Pratheeshkumar P, Son YO, Divya SP, Roy RV, Hitron JA, Wang L, Kim D, Dai J, Asha P, Zhang Z, et al: Luteolin inhibits $\mathrm{Cr}(\mathrm{VI})$-induced malignant cell transformation of human lung epithelial cells by targeting ROS mediated multiple cell signaling pathways. Toxicol Appl Pharmacol 281: 230-241, 2014.

47. Xia F, Wang C, Jin Y, Liu Q, Meng Q, Liu K and Sun H: Luteolin protects HUVECs from TNF- $\alpha$-induced oxidative stress and inflammation via its effects on the Nox4/ROS-NF- $k$ B and MAPK pathways. J Atheroscler Thromb 21: 768-783, 2014. 